\title{
PRIMORDIAL BLACK HOLES AND COSMOLOGICAL PROBLEMS
}

\author{
Alexander Dolgov ${ }^{a}$ \\ Novosibirsk State University, Novosibirsk, 630090, Russia \\ ITEP, Moscow, 117218 Russia
}

\begin{abstract}
.
It is argued that the bulk of black holes $(\mathrm{BH})$ in the universe are primordial $(\mathrm{PBH})$. This assertion is strongly supported by the recent astronomical observations, which allow to conclude that supermassive BHs with $M=\left(10^{6}-10^{9}\right) M_{\odot}$ "work" as seeds for galaxy formation, intermediate mass BHs, $M=\left(10^{3}-10^{4}\right) M_{\odot}$, do the same job for globular clusters and dwarf galaxies, while black holes of a few solar masses are the constituents of dark matter of the universe. The mechanism of PBH formation, suggested in 1993, which predicted such features of the universe, is described. The model leads to the log-normal mass spectrum of PBHs, which is determined by three constant parameters. With proper adjustment of these parameters the above mentioned features are quantitatively explained. In particular, the calculated density of numerous superheavy BHs in the young universe, $z=5-10$, nicely fits the data. The puzzling properties of the sources of the LIGO-discovered gravitational
\end{abstract} waves are also naturally explained assuming that these sources are PBHs.

\section{Introduction}

Recent, and not only recent, astronomical observations revealed many mysterious features of the universe, which were not expected in frameworks of conventional cosmology and astrophysics. All these problems are neatly solved if practically all black holes $(\mathrm{BH})$ in the universe are primordial ones with a wide spread mass spectrum. Primordial black holes by definition are those which were formed in the universe at prestellar epoch, i.e. before stars appeared in sky. The mechanism of their formation was suggested by Zeldovich and Novikov (ZN) 11. According to them, PBH was formed if the density fluctuation in the early universe was of order unity, $\delta \rho / \rho \sim 1$, at the cosmological horizon scale. In this case the piece of space happened to be inside its gravitational radius, so it decoupled from the overall Hubble expansion and a black hole appeared. In the original version $\mathrm{PBH}$ created by such mechanism were rather light (a small fraction of the solar mass) and had narrow (delta-function) mass spectrum. At least such form of the spectrum was mostly assumed in subsequent analysis of observational manifestation of such PBHs.

In 1993 2] a generalization of ZN mechanism was proposed (see also later work [3]), which could lead to very massive PBH with log-normal mass spectrum:

$$
\frac{d N}{d M}=\mu^{2} \exp \left[-\gamma \ln ^{2}\left(M / M_{0}\right)\right],
$$

\footnotetext{
${ }^{a}$ E-mail: dolgov@fe.infn.it
} 
with only 3 parameters: $\mu, \gamma, M_{0}$. The form of the spectrum is practically universal. It is completely determined by the exponential cosmological expansion during inflationary stage.

Fitting the parameters of distribution (1) one can explain the accumulated astronomical data about black holes in contemporary and young universe. In this sense the surprising results of the new precise observations performed in the recent decade are predicted in the papers [2,3].

In this talk I briefly review the following observational data.

Young universe, $z \approx 5-10$, overpopulated by:

1. Bright quasi stellar objects (QSO), super-massive BHs.

2. Superluminous young galaxies.

3. Supernovae and gamma-bursters.

4. Very high level of dust.

Contemporary universe:

1. Supermassive $\mathrm{BH}(\mathrm{SMBH})$ in every large galaxy.

2. SMBH in small galaxies and in almost empty space.

3. Stars older than the Galaxy and even older than the Universe.

4. MACHOs (low luminosity solar mass objects).

5. Problems with the $\mathrm{BH}$ mass spectrum in the Galaxy: unexpected maximum at $M \sim 8 M_{\odot}$.

6. Problems with the sources of the observed gravitational waves (GW).

7. Intermediate mass, $\geq 10^{3} M_{\odot}$, BHs in globular clusters and dwarf galaxies. More details and references can be found in [4, 5 .

\section{Young universe}

\section{1 $S M B H$}

About 40 quasars with $z>6$ are already known, each quasar containing SBH with $M \sim 10^{9} M_{\odot}$. The maximum redshift value among these quasars reaches $z=7.085$ i.e. the quasar was formed before the universe reached 0.75 Gyr. Its luminosity and mass are respectively $L=6.3 \cdot 10^{13} L_{\odot}$ and $M=2 \cdot 10^{9} M_{\odot} 6$. Other high $z$ quasars have similar properties. The formation of SMBHs, which fuel these quasars through the standard accretion mechanism, demands much more time than the universe age at $z \sim 6$. The unsolvable problem with creation of these SMBHs was multiply deepened with the discovery of a real "monster" of 12 billions solar masses [7, i.e. an order of magnitude more massive, than the mentioned above forty. Even the formation of the contemporary SMBH, which had in their disposal the whole universe age, $14 \mathrm{Gyr}$, is difficult to explain, see the next section.

After this Conference was over, a new discovery of a SMBH at now new maximum redshift $z \approx 7.5$ and the mass 0.8 billion solar masses was announced 8 . This is not the largest mass in the family of high redshift quasars, but what 
makes it particularly interesting is that the surrounding matter is neutral, not ionized. This is a very strong argument against formation of this SMBH by the usual accretion process, so its primordial origin remains the only natural possibility.

\subsection{Early bright galaxies}

Several galaxies have been observed at high redshifts, with natural gravitational lens "telescopes". A few examples are:

1) A galaxy at $z \approx 9.6$ which was created when the universe was younger than 0.5 Gyr 9 .

2) A galaxy at $z \approx 11$ [10, which already existed when the universe age was $t_{U} \sim 0.4 \mathrm{Gyr}$. It is particularly impressive that this very young galaxy is three times more luminous in UV than other galaxies at $z=6-8$. This is a striking example of unexpectedly early burn and powefull creature.

3) Not so young but extremely luminous galaxy was found three years ago. Its luminosity reaches gigantic magnitude, $L=3 \cdot 10^{14} L_{\odot}$. The universe age when the galaxy already existed was $t_{U} \sim 1.3$ Gyr. According to the authors of the discovery: "The new study outlines three reasons why the black holes in the extremely luminous infrared galaxies, could have grown so massive. First, they may have been born big. In other words, the galactic seeds, or embryonic black holes, might be bigger than thought possible." One of the authors, P. Eisenhardt said: "How do you get an elephant? One way is start with a baby elephant." The BH was already billions of $M_{\odot}$, when our universe was only a tenth of its present age of 13.8 billion years. "Another way to grow this big is to have gone on a sustained binge, consuming food faster than typically thought possible." For the realization of these conditions low spin is necessary!

According to the paper "Monsters in the Dark" [1] density of galaxies at $z \approx 11$ is $10^{-6} \mathrm{Mpc}^{-3}$, an order of magnitude higher than estimated from the data at lower z. Origin of these galaxies is unclear.

These data strongly support the idea that initially primordial SMBHs appeared and later galaxies were seeded by these PBHs. To the best of my knowledge this idea was first pronounced in ref. 2] and the recent observations do confirm the early creation of very massive black holes.

\subsection{Early miscellanea}

The universe at $z=5-10$ was filled with supernovae, gamma-bursters, and was very dusty. To make dust a long succession of events is necessary: first, supernovae exploded to deliver heavy elements into space (metals), then metals cool and form molecules, and lastly molecules make macroscopic pieces of matter. Abundant dust is observed in several early galaxies, e.g. in HFLS3 at $z=6.34$ [12] and in A1689-zD1 [13] at $z=7.55$. The second galaxy is the 
earliest one where interstellar medium is observed The universe age at this redshift is below 0.5 Gyr.

Catalogue of the observed dusty sources 14 indicates that their number is an order of magnitude larger than predicted by the canonical theory of galaxy evolution.

Hence, prior to or simultaneously with the QSO formation a rapid star formation should take place. These stars should evolve to a large number of supernovae enriching interstellar space by metals through their explosions which later make molecules and dust. (We all are dust from SN explosions, but probably at much later time.)

Observations of high redshift gamma ray bursters (GBR) also indicate a high abundance of supernova at large redshifts. The highest redshift of the observed GBR is 9.4 and there are a few more GBRs with smaller but still high redshifts. The necessary star formation rate for explanation of these early GBRs is at odds with the canonical star formation theory.

\section{Mysteries in the sky today and in the nearest past}

\subsection{Supermassive black holes}

Every large galaxy and some smaller ones contain a central supermassive BH with mass typically larger than $10^{9} M_{\odot}$ in giant elliptical and compact lenticular galaxies, and $\sim 10^{6} M_{\odot}$ in spiral galaxies like Milky Way. The origin of these $\mathrm{BHs}$ is unclear. The accepted faith is that these BHs are created by the matter accretion to galactic center with an excessive mass density. However, the usual accretion efficiency is insufficient to create them during the Universe life-time, $t_{U} \approx 14$ Gyr. Even more puzzling is that SMHBs are observed in small galaxies and even in almost empty space, where no material to make a $\mathrm{SMBH}$ can be found.

Below several examples are presented demonstrating serious inconsistencies between observation and theoretical picture.

The mass of BH is typically $0.1 \%$ of the mass of the stellar bulge of galaxy but some galaxies may have huge BH: e.g. NGC 1277 has the central BH of $1.7 \times 10^{10} M_{\odot}$, or $60 \%$ of its bulge mass $[15$. This creates serious problems for the standard scenario of formation of central supermassive BHs by accretion of matter in the central part of a galaxy.

According to ref. 16, the galaxies, Henize 2-10, NGC 4889, and NGC1277 are examples of SMBHs at least an order of magnitude more massive than their host galaxy suggests. The dynamical effects of such ultramassive central black holes are unclear.

A recent discovery [18] of an ultra-compact dwarf galaxy older than $10 \mathrm{Gyr}$, enriched with metals, and probably with a massive black hole in its center also seems to be at odds with the standard model. 
In the paper entitled "An evolutionary missing link? A modest-mass earlytype galaxy hosting an over-sized nuclear black hole" [17, a black hole with the mass $M_{B H}=(3.5 \pm 0.8) \cdot 10^{8} M_{\odot}$, is found inside the host galaxy with mass of the stars $M_{\text {stars }}=2.5_{-1.2}^{+2.5} \cdot 10^{10} M_{\odot}$, and huge accretion luminosity: $L_{A G N}=(5.3 \pm 0.4) \cdot 10^{45} \mathrm{erg} / \mathrm{s} \approx 10^{12} L_{\odot}$, equal to $12 \%$ of the Eddington luminosity. The active galactic nuclei (AGN) is more prominent than expected for a host galaxy of this modest size. The data are in tension with the accepted picture in which this galaxy would recently have transformed from a star-forming disc galaxy into an early-type, passively evolving galaxy.

Probably the most impressive in this list is a discovery of "A Nearly Naked Supermassive Black Hole" 19. According to the paper, a compact symmetric radio source B3 $1715+425$ is too bright (brightness temperature $\sim 3 \times 10^{10} \mathrm{~K}$ at observing frequency $7.6 \mathrm{GHz})$ and too luminous $\left(1.4 \mathrm{GHz}\right.$ luminosity $\sim 10^{25}$ $\mathrm{W} / \mathrm{Hz}$ ) to be powered by anything but a $\mathrm{SMBH}$, but its host galaxy is much smaller.

There are more example of such puzzling galaxies with superheavy black holes but even with the presented ones the inverted picture of galaxy formation looks more plausible, when first a supermassive black hole was formed and later it attracted matter serving as a seed for subsequent galaxy formation.

\section{2 $\mathrm{MACHOs}$}

MACHO is the name of some invisible or low luminosity objects discovered through gravitational microlensing by Macho [20,21] and Eros [22] groups respectively in the Galactic halo and in the direction to the center of the Galaxy. Later they were registered in the Andromeda (M31) galaxy [23]. The masses of the registered objects are about one half of the solar mass. The up to date situation with MACHOs is summarized in ref. 24:

Macho group: $0.08<f<0.50(95 \% \mathrm{CL})$ for $0.15 M_{\odot}<M<0.9 M_{\odot}$;

EROS: $f<0.2,0.15 M_{\odot}<M<0.9 M_{\odot}$;

EROS2: $f<0.1,10^{-6} M_{\odot}<M<M_{\odot}$;

AGAPE: $0.2<f<0.9$, for $0.15 M_{\odot}<M<0.9 M_{\odot}$;

EROS-2 and OGLE: $f<0.1$ for $M \sim 10^{-2} M_{\odot}$ and $f<0.2$ for $\sim 0.5 M_{\odot}$.

Thus, the MACHO density is comparable to the density of the halo dark matter but their nature is unknown. They could be brown dwarfs, dead stars, or primordial black holes. The first two options are in conflict with the accepted theory of stellar evolution, if such invisible stars were created in the conventional way.

The only remaining option is that MACHOs are low mass black holes, but one can hardly imagine that such low mass black holes, abundant in the Galactic halo, were created as a result of stellar collapse of normal stars. So the natural conclusion is that MACHOs are primordial black holes as it is stated in ref. 3. The log-normal spectrum of the $\mathrm{PBH}$ allows to make much larger contribution 
to DM from heavier PBH, to which the microlensing method is not sensitive. So ultimately $100 \%$ of DM may be made out of PBHs with different masses.

\subsection{Properties of the sources of gravitational waves}

Direct registration of gravitational waves (GW) by LIGO 25] revealed intriguing properties of the GW sources [26. The shape of the signal in the interferometer is well described by the assumption that the observed GWs are produced by the binary of coalescing BHs, but:

1. The origin of heavy BHs with masses $\sim 30 M_{\odot}$ is unclear. Such BHs are believed to be created by massive star collapse, though a convincing theory is still lacking. To form so heavy BHs, the progenitors should have $M>100 M_{\odot}$ and a low metal abundance to avoid too much mass loss during the evolution. Such heavy stars might be present in young star-forming galaxies but they are not yet observed in sufficiently high number.

2. In all events, but one, the spins of the coalescing BHs are very small. compatible with zero. It strongly constrains astrophysical $\mathrm{BH}$ formation from close binary systems. However, the dynamical formation of double massive low-spin BHs in dense stellar clusters is not excluded, but difficult.

3. Formation of BH binaries from the original stellar binaries has very low probability. Stellar binaries were formed from common interstellar gas clouds and are quite frequent in galaxies. If $\mathrm{BH}$ is created through stellar collapse, a small non-sphericity of the collapse results in a huge velocity of the $\mathrm{BH}$ and the binary is destroyed. BH formation from PopIII stars and subsequent formation of $\mathrm{BH}$ binaries with $(36+29) M_{\odot}$ is analyzed and found to be negligible.

All these problems are solved if the observed sources of GWs are the binaries of primordial black holes (PBH).

\subsection{Globular clusters and intermediate mass BHs.}

Recently the so called intermediate mass black holes (IMBH) with masses $M \approx 2000 M_{\odot}$ and $M \sim 20000 M_{\odot}$ were presumably observed in the centers of globular clusters 27, 28. These observations nicely fit our conjecture 29] that IMBH play an important role in the formation and evolution of globular clusters. Using the parameters of the mass distribution (11), found in our paper [26], we find that the density of the primordial IMBH is sufficient to seed the formation of all globular clusters observed in galaxies. 
In addition to globular clusters, IMBHs are probably also contained in centers of dark stellar clusters 30,31. These clusters have high mass-luminosity ratio. They may be the remnants of dwarf spheroids with the masses between those of globular clusters and large galaxies. It looks natural that these spheroids were seeded by the primordial IMBH [29].

\subsection{Solar mass Black holes in the Milky Way}

The mass spectrum of black holes observed in the Galaxy demonstrates some peculiar features, which are difficult to explain in the standard model of $\mathrm{BH}$ formation by stellar collapse. In particular, it is found 32 that the masses of black holes in the Galaxy are concentrated in the narrow range $(7.8 \pm 1.2) M_{\odot}$. This result agrees with another paper where a peak around $8 M_{\odot}$, a paucity of sources with masses below $5 M_{\odot}$, and a sharp drop-off above $10 M_{\odot}$ are observed 33.

On the other hand, such mass spectrum is well described by the log-normal form. This is an argument in favor of primordial origin of the black holes in the Galaxy.

\subsection{Old stars in the Milky Way}

Recently several groups presented substantially more accurate determinations of stellar ages in the Galaxy. Surprisingly quite a few stars happened to be considerably older than expected.

According to ref. [34]: employing thorium and uranium abundances in comparison with each other and with several stable elements the age of metal-poor, halo star $\mathrm{BD}+17^{\circ} 3248$ was estimated as $13.8 \pm 4 \mathrm{Gyr}$. This star is much older than the inner halo of the Galaxy, which has the age equal to $11.4 \pm 0.7$ Gyr [35].

The age of another star in the galactic halo, HE 1523-0901, was estimated to be about 13.2 Gyr [36]. First time many different chronometers, such as the $U / T h, U / I r, T h / E u$, and $T h / O s$ ratios to measure the star age, have been employed.

And at last a star older than the universe was found [37. Metal deficient high velocity subgiant in the solar neighborhood HD 140283 has the age $14.46 \pm 0.31$ Gyr. The central value exceeds the universe age by two standard deviations, if $H=67.3$, and $t_{U}=13.8 \mathrm{Gyr}$; while if $H=74$, and $t_{U}=12.5 \mathrm{Gyr}$, the star would be older than the universe by more than $10 \sigma$. This is of course impossible, but the star may look older that it is, if initially the star was enriched by heavy elements and evolve to its present state faster than the normal one. Our model of PBH formation [2,3] leads also to creation of compact primordial stellar-like objects consisting not only from hydrogen and helium but enriched with plenty of heavier elements. 


\section{Mechanism of massive PBH formation}

In this Section the main features of the mechanism [2,3] of massive PBH formation are described. We assume that a slightly modified baryogenesis scenario suggested by Affleck and Dine (AD) [38] is realized. The main ingredient of this $\mathrm{AD}$-scenario is a scalar field $\chi$ with non-zero baryonic number $B$. It is assumed that the potential of $\chi$ has the so called flat directions along which the potential does not rise. In the course of the cosmological expansion $\chi$ might acquire large expectation value turning practically into a classical field with large $B$. Later after decay of $\chi$ this accumulated baryonic number turned into baryonic number of quarks, leading to a large baryon asymmetry $\beta$ of the universe. It may be even of order unity, while the observed value of $\beta$ is about $10^{-9}$,

We modified the AD mechanism by introduction of general renormalizable coupling of $\chi$ to the inflaton field $\Phi$ (the first term in the r.h.s. of the equation below), which can be written in the form:

$$
U=g|\chi|^{2}\left(\Phi-\Phi_{1}\right)^{2}+\lambda|\chi|^{4} \ln \left(\frac{|\chi|^{2}}{\sigma^{2}}\right)+\lambda_{1}\left(\chi^{4}+\text { h.c. }\right)+\left(m^{2} \chi^{2}+\text { h.c. }\right) .
$$

With this interaction the flat direction of the potential $U$ are open only when $\Phi \approx \Phi_{1}$, which was taken by $\Phi$ in the course of inflation before it was over. If the window to flat direction, when $\Phi \approx \Phi_{1}$ is open only during a short period, cosmologically small but possibly astronomically large bubbles with high $\beta$ could be created, These bubbles with large $\beta$ might occupy only a small fraction of the universe volume, while the rest of the universe would have the normal small baryon-to-photon ratio $\beta \approx 6 \cdot 10^{-10}$, created by small $\chi$, which did not succeed to penetrate through the briefly open window to a large value.

After the QCD phase transition, when massless quarks turned into heavy nucleons, the initial isocurvature perturbation created by inhomogeneities in the chemical content turned into (large) density perturbations. This would lead to an early formation of $\mathrm{PBH}$ or compact stellar-type objects with high baryonic density. As a result, the bulk of baryons and maybe antibaryons would be contained in compact cosmologically tiny stellar-like objects or PBH. These high-B density bubbles would live in huge by size but not so dense universe with low baryonic background density, which initially was practically homogenenous.

The formation of PBHs or compact stellar type objects took place at very high $z$ after the QCD phase transition at $T \sim 100 \mathrm{MeV}$ down to $T \sim \mathrm{keV}$.

As a byproduct, the mechanism of refs. 23] may lead, though not necessarily, to abundant compact antimatter objects in the universe and, in particular, in the Galaxy [24,39,40]. 


\section{Conclusion}

The problems emerged from the multitude of astronomical observations, some of which are mentioned in this talk, are uniquely and simply resolved if the universe is populated by the primordial massive black holes and stellar-like compact objects with wide mass spectrum. The mechanism which leads to an abundance of such objects in the universe was put forward in 1993 [2, 3] and essentially predicted the subsequent surprising discoveries.

All the multitude of the various astronomical data are well explained by the natural baryogenesis model which leads to formation of PBHs and compact stellar-like objects in the early universe after the QCD phase transition, $t \leq 10^{-5}$ sec. These objects are predicted to have log-normal mass spectrum. They can be numerous enough to give significant contribution to the cosmological dark matter or even make all of it.

The model opens the possibility for the inverted picture of the galaxy formation, when firstly supermassive black holes are formed which later accrete matter creating galaxies. The new observations persuasively indicate in this direction. Lighter PBHs with $2000 M_{\odot}$ are predicted in sufficient amount to explain the origin of globular clusters, while heavier PBHs, with $M \sim 10^{4} M_{\odot}$ can seed formation of dwarf spheroids. There seem to be strong indications in favor of this scenario.

PBHs formed through such mechanism can explain the peculiar features of the sources of GWs observed by LIGO.

The considered mechanism resolves the numerous mysteries of $z \sim 10$ universe: abundant population of supermassive black holes, early created gammabursters and supernovae, early bright galaxies, and evolved chemistry including dust.

Existence of high density invisible "stars" (MACHOs) is explained.

"Older than $t_{U}$ " stars may exist. The old age is mimicked by the unusual initial chemistry.

The model can possibly lead to the prediction of numerous compact antimatter objects (antistars). The observational data allow for large amount of such objects in the Galaxy. However, their density is model dependent and the prediction is uncertain.

\section{Acknowledgments}

This work was supported by RSF Grant No. 16-12-10037.

[1] Ya.B. Zeldovich, I.D. Novikov, Sov. Astron. 10, 602 (1967).

[2] A. Dolgov, J. Silk, Phys.Rev., D47 (1993), 4244.

[3] A.D. Dolgov, M. Kawasaki, N. Kevlishvii, Nucl. Phys., B807 (2009), 229. 
[4] A.D. Dolgov, e-Print: arXiv:1605.06749.

[5] A.D. Dolgov, e-Print: arXiv:1701.05774.

[6] D.J. Mortlock, et al, Nature 474 (2011) 616; arXiv:1106.6088.

[7] Xue-BingWu et al, Nature 518, 512 (2015).

[8] E. Bañados, et al, arXiv:1712.01860v1

[9] W. Zheng, et al, Nature, 489 (2012) 406; arXiv:1204.2305,

[10] D. Coe et al Astrophys. J. 762 (2013) 32.

[11] D. Waters, et al, Mon. Not. Roy. Astron. Soc. 461 (2016), L51.

[12] Clements, D.L., et al, arXiv:1511.03060.

[13] Mattsson T., arXiv:1505.04758.

[14] Asboth V., et al, arXiv:1601.02665.

[15] Bosch van den R. C. E., et al., Nature, 491 (2012), 729.

[16] F. Khan, K. Holley-Bockelmann, P. Berczik arXiv:1405.6425,

[17] J. Th. van Loon, A.E. Sansom, arXiv:1508.00698v1.

[18] J. Strader, et al Ap. J. Lett. 775, L6 (2013).

[19] J.J. Condon, et al arXiv:1606.04067,

[20] Alcock A., et al, Astrophys. J., 542 (2000), 281.

[21] Bennett D.P., Astrophys. J. 633 (2005), 906.

[22] Tisserand P., et al, A\&A, 469 (2007), 387.

[23] Riffeser A., Seitz S., Bender R., Astrophys. J., 684 (2009), 1093.

[24] S.I. Blinnikov, A.D. Dolgov, K.A. Postnov, Phys. Rev., D92 (2015), 023516 .

[25] Abbott B.P., et al., Phys. Rev. Lett., 116 (2016), 061102.

[26] S. Blinnikov, et al, JCAP, 1611 (2016), 036.

[27] Kiziltan B., Baumgardt H., Loeb A., Nature, 542 (2017,) 203

[28] Perera B.B.P., et al., arXiv:1705.01612,

[29] A. Dolgov, K. Postnov, JCAP, 1704 (2017), 036.

[30] M. A. Taylor M.A., et al., Astrophys. J., 805 (2015), 65, [1503.04198].

[31] M. S. Bovill, M.S., et al., Astrophys. J., 832 (2016), 88; [1608.06957].

[32] F. Ozel F., et al., arXiv:1006.2834

[33] L. Kreidberg, et al, arXiv:1205.1805.

[34] J.J. Cowan, et al Ap.J. 572 (2002) 861

[35] J. Kalirai, Nature 486 (2012) 90, arXiv:1205.6802,

[36] A. Frebe, et al Astrophys. J. 660 (2007) L117.

[37] H. E. Bond, et al, Astrophys. J. Lett. 765, L12 (2013), arXiv:1302.3180

[38] Affleck I., Dine M., Nucl. Phys. B 249 (1985), 361.

[39] C. Bambi, A. D. Dolgov, Nucl. Phys. B 784, 132 (2007).

[40] A. D. Dolgov, S. I. Blinnikov, Phys. Rev. D 89, 021301(R) (2014). 